\title{
Language Assessment in Aphasia: An international survey of practice
}

Julie Morris and Janet Webster. Speech and Language Sciences, School of Education, Communication and Language Sciences, Newcastle University, Newcastle upon Tyne, NE1 7RU, UK

julie.morris@ncl.ac.uk

\section{Background}

Assessment is an integral part of aphasia management, and one of the top ten best practice recommendations proposed by Simmons-Mackie and colleagues (2017): “Assessment should extend beyond the use of screening measures to determine the nature, severity and personal consequences of the suspected communication deficit” (pp. 139). Whilst assessment needs to extend beyond language, assessment of language is nevertheless a key aspect. There are many assessments, including those which consider several aspects of language (e.g. BDAE: Boston Diagnostic Aphasia Examination, CAT: Comprehensive Aphasia Test, PALPA: Psycholinguistic Assessment of Language Processing in Aphasia) and those assessing specific language areas (e.g. of word retrieval).

\begin{abstract}
Aims
This study aimed to understand assessment practice within Speech and Language Therapy/Pathology, exploring use of formal and informal assessments, rationale underpinning assessment use and how clinicians use of some of the commonly reported assessments, which have a broad focus on language.
\end{abstract}

\section{Methods and Procedures}


This study used an online survey to explore assessment, with the link to the survey distributed via professional organisations, including international aphasia organisations and academic networks. Respondents were anonymous, with data collected about the setting, geographical location and experience, followed by a detailed set of questions about assessment practice. Questions included closed questions, some with options or ratings provided and also the opportunity to provide more qualitative information. In addition to generic questions about assessment, respondents were asked to consider the role of three of the major tests in more detail (BDAE, CAT and PALPA).

\section{Outcomes and Results}

243 individuals completed the survey although not everyone answered each question. Respondents were predominantly clinicians working across acute, inpatient, outpatient and/or private settings $(88 \%)$ and a small number of researchers. Respondents were from a range of countries, including UK ( $\mathrm{n}=166)$, Australia (54), Ireland (21) and also including Finland, Germany, USA Singapore, and Jersey. People had a range of years of experience with aphasia, but were predominantly more experienced (5+ years of experience with aphasia).

People reported using both formal and informal assessments; 65\% sometimes, and 34\% always. When this was further explored, as is shown in Figure 1, the majority felt a combination of formal and informal assessment provided the best information. People did not express a preference for one assessment type.

\section{Figure 1 about here}

Asked in detail about the three major assessments, value was placed on their contribution (particularly CAT and PALPA) to diagnosis, goal setting and treatment planning and evaluating outcome. Less value was placed on their role in determining prognosis. 
Within open comments, several responders discussed the need for flexibility, particularly when working in the acute setting. Some respondents also commented on the need for assessments which were appropriate for people with severe aphasia. However, there were several comments expressing a preference for informal assessment and also comments about a restricted range of assessments being available, and about time pressures restricting the assessment carried out.

\section{Conclusions}

It is crucial to understand current assessment practice in aphasia. This survey demonstrates a range of language assessment practice, across settings and countries, with clinicians selecting both formal and informal assessments and using them to inform management in different ways. Qualitative comments reinforce that clinicians are using assessments selectively, often according to constraints of setting and/or time, perceived value of assessment but also importantly the clients' needs.

It is important we continue to explore assessment practice, considering the purpose of assessment and what tool(s) are best suited to the purpose. As Frith et al (2014) discuss, given the variation in practice, there is a need to further enhance understanding to ensure best practice in assessment.

\section{References}

Frith, M., Togher, L., Ferguson, A., Levick, W., \& Docking, K. (2014). Assessment practices of speech-language pathologists for cognitive communication disorders following traumatic brain injury in adults: An international survey. Brain Injury, 28(13-14), 1657-1666. doi:10.3109/02699052.2014.947619 
Goodglass, H., Kaplan, E., \& Barresi, B. (2001). BDAE-3: Boston Diagnostic Aphasia Examination (3rd ed.): LinguiSystems.

Kay, J., Lesser, R., \& Coltheart, M. (1992). Psycholinguistic Assessments of Language Processing in Aphasia (PALPA). Hove: Lawrence Erlbaum Associates.

Simmons-Mackie, N., Worrall, L., Murray, L. L., Enderby, P., Rose, M. L., Paek, E. J., . . Advisory, C. (2017). The top ten: best practice recommendations for aphasia. Aphasiology, 31(2), 131-151. doi:10.1080/02687038.2016.1180662

Swinburn, K., Porter, G., \& Howard, D. (2004). The Comprehensive Aphasia Test. Hove: Psychology Press. 